\title{
C omportamiento diario y estacional de las estructuras óptica y térmica en un embalse tropical colombiano
}

\author{
J ohn J . Ramírez R. \\ Grupo de Limnología Básica y Experimental y Biología y Taxonomía Marina, \\ Facultad de Ciencias Exactas y Naturales, Universidad de Antioquia, Colombia
}

\begin{abstract}
Resumen
Esta investigación se preguntó sobre la variación temporal en las escalas diaria y estacional (anual) de las estructuras óptica y térmica en la presa del embalse de La Fe. Se preveía que la estructura óptica tendría poca variación tanto en la escala diaria como en la anual. En cuanto a la estructura térmica, la hipótesis era que tendría una mayor variación en la escala diaria que en la estacional. Con el propósito de corroborar estas presunciones, se realizaron diez muestreos nictémeros en la columna de agua de la presa a intervalos de cuatro horas durante diez meses (diciembre de 2002 a noviembre de 2003). Las variables evaluadas fueron: temperatura del aire y del agua, velocidad y dirección del viento, profundidad Secchi, profundidad de mezcla, color aparente y turbidez, al igual que los perfiles de temperatura, luz, conductividad eléctrica, oxígeno disuelto, $\mathrm{CO}_{2}$ total y $\mathrm{pH}$. La forma óptica presentó diferencias levemente significativas en la escala diaria y no significativas en la estacional. Por razones como la intensidad del color, el cinturón litoral de Egeria densa, las floraciones de algas, los aportes alóctonos y el patrón atelomíctico del sistema, dicha forma puede ajustarse al tipo GA. Dado que el viento no incidió lo suficiente para desestabilizar la columna de agua, esta se mantuvo estratificada a lo largo del tiempo de muestreo, mezclándose solo en los primeros metros como se espera en un embalse atelomíctico en el que la mezcla se debe principalmente a fuerzas convectivas. En consecuencia, la temperatura del fondo varió muy poco, el perfil de $\mathrm{pH}$ tendió a disminuir, y la conductividad eléctrica y el $\mathrm{CO}_{2}$ total, a aumentar.
\end{abstract}

Palabras clave: represa tropical, estructuras y óptica térmica, dinámica diaria y estacional.

Daily and seasonal behavior of optical and thermical structures in a Colombian tropical reservoir (L a Fereservoir)

\begin{abstract}
The objective of this research was to find out about the temporal variation of optical and thermal structures in $\mathrm{LaFe}$ dam both in daily and seasonal scales. Our hypothesis on the optical structure was that there would be little variation in both time scales. As for the thermal structure, we predicted that it would vary more on a daily basis that in the seasonal scale. In order to corroborate these assumptions, we took ten nictemeral samples in the water column of the dam at four hour intervals during 10 months (December 2002 to November 2003). We evaluated the following variables: air and water temperature, wind direction and speed, Secchi depth, mixing depth, apparent color and turbidity, and profiles of electrical conductivity, dissolved oxygen, total $\mathrm{CO}_{2}$ and $\mathrm{pH}$. The optical shape presented slightly significant differences on the daily scale and non-significant differences in the seasonal scale. Given its intense color, littoral belt of Egeria densa, algal blooms, allochthonous inputs, and the atelomictic pattern of the system, its shape may be considered as being a GA type. As the wind didn't work long enough to destabilize the water column, it remained stratified all along the sampling period and mixture was present only in the first few meters as can be expected in an atelomictic reservoir in which the mixture is mainly due to convective forces. Consequently, bottom temperature varied very little, the $\mathrm{pH}$ profile tended to decrease, and the electrical conductivity and total $\mathrm{CO}_{2}$ to increase.
\end{abstract}

Key words: Tropical dam, optical and thermal structures, diurnal and seasonal dynamics.

\section{Introducción}

Los ritmos diarios o nictémeros se asocian a ritmos endógenos de respuesta que no sólo se relacionan con conductas periódicas vitales, sino también con el efecto de la oscilación en la mayoría de los factores externos como luz, la radiación y la temperatura, en el ciclo día-noche (M argalef, 1974). La duración del día y de la noche es función de la latitud y del momento del año. En las zonas templadas dicha variación es alta, en las ecuatoriales ( 0 a $10^{\circ}$ de latitud norte y sur) y en las tropicales ( 10 a $23^{\circ}$ de latitud norte y sur) existe

\section{Correspondencia:}

John Jairo Ramírez R., johnra77@gmail.com

Recibido: 27 de noviembre de 2014

A ceptado: 15 de marzo de 2015 
una mayor constancia en la duración del día y la noche y, puesto que la longitud del día varía muy poco (22 minutos entre el día más largo y el más corto del año y 2,4 ${ }^{\circ} \mathrm{C}$ entre el día más cálido y el más frío en el valle del río Magdalena), no es común referirse a fotoperiodo. Sin embargo, diversos estudios comparativos entre la variación diaria y la mensual han llevado a concluir que en las zonas de latitudes bajas la variación nictémera en estas escalas es muy superior a la que se observa en la escala anual, considerándose por ello que los ecosistemas lacustres en estas zonas están dominados por un ciclo de 24 horas más que por uno estacional ( $G$ anf \& Horne, 1975).

Como sistema termodinámico abierto, un lago natural o artificial posee entre otros atributos los siguientes: 1) una forma óptica originada en el flujo neto de cuantos producto del balance entre el ingreso de energía electromagnética sin trasporte de materia y las pérdidas en dicho balance ocasionadas por la reflexión, la refracción, la absorción, la dispersión y la extinción, y 2) una forma térmica causada por el balance entre las fuerzas viscosas estabilizadoras originadas en los flujos de calor (mediante los afluentes y la radiación solar) y su pérdida (a través de los efluentes, la convección y la mezcla). En este tipo de sistemas la estabilidad se alcanza cuando las tasas de cambio entre componentes y la velocidad a la que mudan se retardan para hacer frente a cualquier intento externo de modificación de las estructuras o formas logradas. Uno de los mecanismos utilizados para conservar esta condición y evitar la inestabilidad es la tendencia a realzar las fronteras con el fin de disipar los efectos de la entropía del entorno (Margalef, 1980; 1993). Una de estas vías es el ‘acomodo’ estructural, tanto óptico como térmico, que se produce frente a las variaciones diarias en la entrada de energía electromagnética. Esta adecuación estructural es equivalente a la incorporación de orden, neguentropía o entropía negativa, representado por la presencia de zonas o límites determinados, como el límite de la zona fótica y la termoclina. La presencia de estos límites, surgidos en el seno mismo del sistema, permite que se mantenga estable y a un nivel bajo de desorden (poca entropía), incorporando continuamente orden desde su medio (alrededores), es decir que, al igual que cualquier objeto material, presentará una estructura mudable, una 'forma', que le agregará propiedades particulares y concretas (Margalef, 1993).

Las investigaciones sobre variación diaria y estacional de la estructura térmica en sistemas lacustres embalsados de Colombia son relativamente pocas (Villegas, 2004; Vergara, 2005; Fernández, 2006; Ramos, 2007; M arín, 2008; Román, 2011; Franco-Velásquez, 2011; Román, et al., 2013, entre otros pocos). Otras publicaciones, la mayoría de ellas basadas en investigaciones ecológicas y biológicas tradicionales, son, entre otras muchas, las de Ramírez \& Díaz (1995a 1995b), Urrego \& Ramírez (2000), Ríos (2005), M ontoya-M oreno (2008) y M ontoyaMoreno \& Aguirre (2009). Ocurre otro tanto con la variación a escalas diaria y anual de la estructura óptica. El estudio de la conducta de este tipo de estructura en los medios acuáticos es objeto de la óptica hidrológica. Según K irk (2011), si se quiere establecer la conducta óptica de un sistema acuático, es necesario medir el grado en que el agua absorbe y dispersa. Los procesos de absorción y dispersión de la luz a cualquier longitud de onda se especifican en términos de las propiedades ópticas inherentes (coeficientes de absorción y dispersión); la suma de los dos constituye la atenuación de la luz en el agua, expresada a través del coeficiente de atenuación vertical para irradiancia descendente $\left(\mathrm{K}_{\mathrm{d}}(\mathrm{PAR})\right.$, según K irk, 2011), que es una expresión cuantitativa de la disminución de una cantidad radiométrica específica con la profundidad.

Así, en esta investigación se buscó responder a la siguiente pregunta: ¿cómo se da la variación temporal diaria y anual de las estructuras óptica y térmica en la columna de agua de la presa del embalse La Fe? Para la estructura óptica se previó que debido a la morfometría del sistema, a los tiempos de retención relativamente altos, al cinturón de macrófitas que en buena parte retiene los sedimentos que ingresan por los afluentes, al poco sombreo de las algas en razón del tamaño relativamente grande de los organismos fitoplanctónicos dominantes, a las condiciones edáficas de la zona donde se ubica el cuerpo de agua, y al tratamiento previo al que son sometidas las aguas del municipio de El Retiro, la estructura óptica es poco variable tanto en la escala diaria como en la anual. Respecto a la estructura térmica se planteó la hipótesis de que si en la zona tropical las variaciones diarias de temperatura son mucho más pronunciadas que las anuales, entonces las estructuras térmicas varían más en esa escala que en la estacional.

\section{$M$ étodos}

\section{Descripción del área}

El embalse de La Fe (Figura 1) fue llenado en 1973 y está a cargo de las Empresas Públicas de Medellín. Sus características principales se presentan en la tabla 1. Es el resultado de dos fases de construcción: la primera, formada por una pequeña presa de gravedad finalizada en 1967, se denominó “proyecto embalse 'Los Salados””; la segunda, producto de una ampliación en 1974, dio origen al actual embalse de La Fe. La antigua presa divide horizontalmente el sistema en dos cuencos: norte y sur. En el cuenco norte (área: 0,68 km², $\mathrm{D}_{\mathrm{m}}: 7,0 \mathrm{~m}, \mathrm{D}_{\text {máx }}: 18,2 \mathrm{~m}$ ), se encuentran la torre de captación y el vertedero y en él tributan los principales afluentes naturales (Palmas y Espíritu Santo); es un sitio de poca profundidad debido a la alta deposición de sedimentos provenientes de los afluentes, a la barrera que genera la antigua presa de Los Salados y a la aducción de la captación que reduce el transporte de sedimentos de los afluentes (Román, 2011; Román, et al., 2013). En su nacimiento, la microcuenca de Las Palmas tiene cultivos de papa y pastos destinados al ganado lechero, en los cuales 


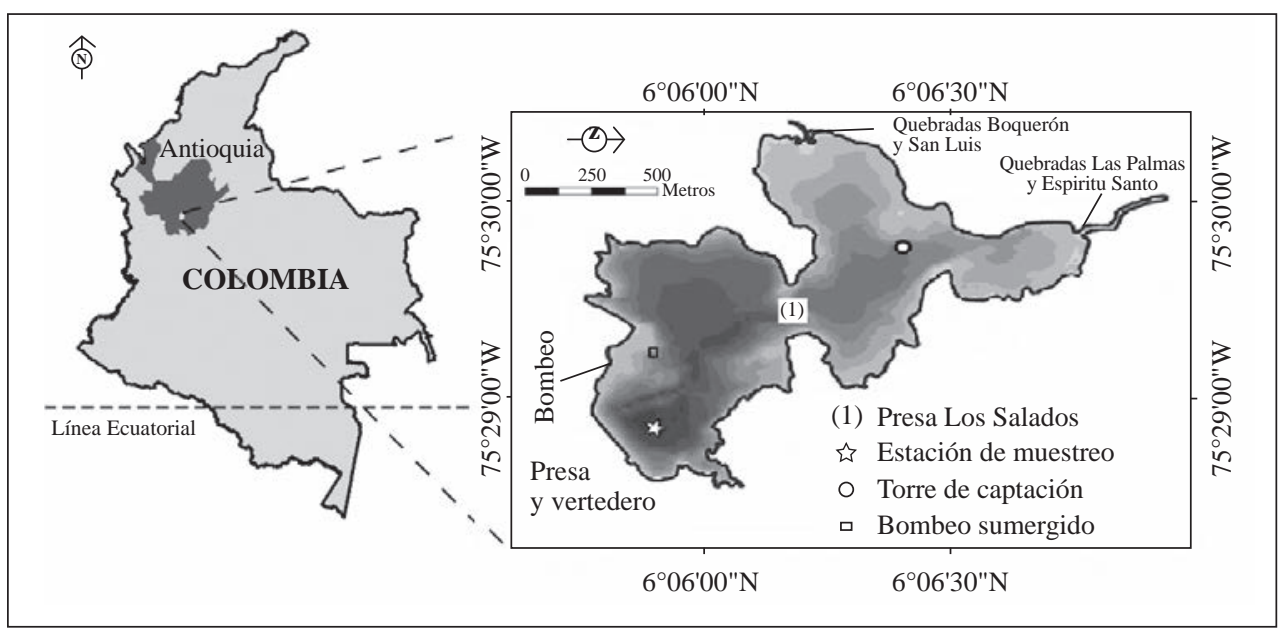

Figura 1. Embalse de La Fe. Mapa orientado de la represa con la ubicación de la estación de muestreo (modificado de R omán, 2011)

Tabla 1. Valores de los principales parámetros morfométricos del embalse de La Fe (Hernani \& Ramírez, 2002)

\begin{tabular}{lc}
\hline Característica & Valor \\
\hline Altitud (cota máxima) & $2155,0 \mathrm{~m}$ \\
\hline Localización & $75^{\circ} 30^{\prime} 15^{\prime}$ O y $06^{\circ} 06^{\prime} 50^{\prime \prime} \mathrm{N}$ \\
\hline Caudal medio extraído & $8,0 \mathrm{~m}^{3} / \mathrm{s}$ \\
\hline Tiempo de retención & $18,0-29,0$ días \\
Área superficial & 143,0 a $173,0 \mathrm{~km}^{2}$ \\
\hline Volumen & 14,0 a $15,0 \mathrm{~mm}^{3}$ \\
Perímetro & $8,0 \mathrm{~km}$ \\
\hline Profundidad media & $9,9 \mathrm{~m}$ \\
Profundidad máxima & $27,0 \mathrm{~m}$ \\
Índice de desarrollo de la línea costera $(\mathrm{F})$ & 2,2 \\
Índice de desarrollo de volumen $\left(\mathrm{D}_{\mathrm{v}}\right)$ & 1,1 \\
Profundidad relativa $\left(\mathrm{D}_{\mathrm{r}}\right)$ & $2,1 \%$ \\
\hline
\end{tabular}

se aplican fertilizantes y plaguicidas; se presenta, además, contaminación por aguas residuales provenientes de las viviendas y zonas de comercio localizadas a corta distancia de las fuentes de agua, las cuales no se someten a tratamiento pues se carece de sistemas de tratamiento de aguas residuales. Alrededor de la quebrada Espíritu Santo predominan los potreros y las fincas de recreo.

En el cuenco sur (área: 0,65 km², $\mathrm{D}_{\mathrm{m}}$ : 14,7 m, $\mathrm{D}_{\text {máx }}$ : = 27,0 $\mathrm{m})$, se ubica la presa del embalse que recibe las aguas del bombeo del río Pantanillo ((3000 1/s) las cuales son tratadas previamente mediante un sistema de lodos activados en la planta de tratamiento localizada en el municipio de El Retiro. El embalse se utiliza primordialmente como acueducto, pero una de sus secciones está destinada a la recreación (Parque Los Salados), y ocasionalmente se utiliza para la generación hidroeléctrica (R omán, 2011; R oman, et al., 2013).

\section{Muestreos}

Se realizaron 10 muestreos nictémeros (diciembre de 2002 a noviembre de 2003) en la presa del embalse a intervalos de cuatro horas, comenzando siempre a las $6 \mathrm{~h} 00$ y terminando a la misma hora del día siguiente.

La temperatura del aire se midió con un termómetro de mercurio (escala Celsius). La velocidad del viento $(\mathrm{m} / \mathrm{s})$ se cuantificó con un anemómetro y su dirección con una veleta de tela, una brújula y una rosa de los vientos. Los datos de dirección se convirtieron a grados sexagesimales.

Para evaluar la estructura óptica se consideró el coeficiente de atenuación vertical para irradiancia descendente $\left(\mathrm{K}_{d}(\mathrm{PAR})\right.$, en $\mathrm{m}^{-1}$ ), entendido como la tasa de pérdida de luz con la profundidad, el cual se obtuvo a partir de los perfiles de luz, los cambios de la transparencia, la profundidad de la zona fótica, el color aparente y la turbidez. Los perfiles de luz $(\mu \mathrm{E} /$ $\mathrm{cm}^{2} / \mathrm{s} 1$ ) se midieron cada $50 \mathrm{~cm}$ con un cuantómetro Licor provisto de un sensor esférico. La transparencia se midió con un disco de Secchi de 0,20 cm de diámetro y bandas blancas y negras alternadas. A partir de dicha medición se multiplicó por 3,0 para estimar la profundidad de la zona fótica. El color aparente se evaluó con un medidor Hach, y la turbidez (NTU) con un turbidímetro de la misma marca.

El perfil térmico se estableció con un termistor YSI cada 50 cm hasta los $10 \mathrm{~m}$ y de allí en adelante cada metro hasta el fondo de la columna de agua. En las mismas profundidades y usando el mismo equipo, se midió la concentración de oxígeno disuelto.

Se seleccionaron los siguientes criterios de evaluación de la estructura o forma térmica: 1) la tasa de cambio de la estructura térmica con la profundidad $\left(\mathrm{TD}_{\text {temp }}\right.$ en $\left.{ }^{\circ} \mathrm{C} / \mathrm{m}\right)$, y 2) la tasa de cambio de la resistencia térmica relativa (RTR) a la mezcla $\left(\mathrm{TD}_{\mathrm{RTR}}\right.$ en $\left.\mathrm{m}^{-1}\right)$. La primera se resumió usando la pendiente de la curva obtenida mediante un análisis de regresión lineal simple $\left[\mathrm{T}^{\circ}=\mathrm{f}\right.$ (profundidad) $]$. Para obtener 
la segunda, se utilizó la diferencia entre la RTR del fondo y la de la superficie dividida por la respectiva diferencia de profundidad (Padisák, et al., 2003). Los valores de la RTR se obtuvieron con el método sugerido por Hutchinson (1957) y Cole (1983). Al igual que la temperatura, la tasa de pérdida de oxígeno disuelto $\left(\mathrm{TDO}_{2}\right.$ en $\left.\mathrm{mgO}_{2} / \mathrm{l} / \mathrm{m}\right)$ se cuantificó mediante una regresión lineal simple $\left\{\left[\mathrm{O}_{2}\right]=\mathrm{f}\right.$ (profundidad) $\}$.

Las variables correlativas que permitieron evaluar la estabilidad de la forma térmica fueron la conductividad eléctrica (conductímetro WTW), el $\mathrm{CO}_{2}$ total (McKereth, et al., 1978) y el pH (medidor de WTW). Los perfiles de estas variables se establecieron extrayendo muestras de agua de siete profundidades en la columna de agua con una botella Schindler ( $=5$ l). Dado que la profundidad de la columna de agua varía de acuerdo a la operación del embalse, dichas profundidades no fueron fijas.

Para estimar el intercambio calórico entre el aire y el agua (convección), se usó la diferencia entre la temperatura del aire, $\mathrm{T}_{\mathrm{a}}$, y la temperatura del agua en la superficie, $\mathrm{T}_{\mathrm{s}}$; dicha diferencia toma valores positivos si $\mathrm{T}_{\mathrm{a}}>\mathrm{T}_{\mathrm{s}}$; de cero cuando $\mathrm{T}_{\mathrm{a}}=\mathrm{T}_{\mathrm{s}}$, o negativos cuando $\mathrm{T}_{\mathrm{a}}<\mathrm{T}_{\mathrm{s}}$. Si el valor negativo es considerable, se generan corrientes convectivas que pueden erosionar la termoclina.

La profundidad de la capa mezclada se definió según la propuesta de L ewis (1987) como aquella porción del perfil térmico que presenta una temperatura uniforme cerca de la salida del sol, antes de la incorporación diaria de calor.
La profundidad relativa (Dr) se calculó según lo propuesto por Hutchinson (1957) y Cole (1983).

En la figura 2 se presentan algunos ejemplos de los perfiles ópticos. Las figuras de los perfiles de los ciclos diarios de temperatura (Figura 3) tienen una línea punteada cuyo objetivo es mostrar el grado de inclinación del metalimnio a lo largo del tiempo de muestreo.

\section{Tratamiento estadístico}

Para el análisis descriptivo se usó la media aritmética como medida de tendencia central. Para evaluar la dispersión absoluta y la relativa se utilizaron la desviación estándar (S) y el coeficiente de variación (CV). Las isotermas se elaboraron usando el programa Surfer, versión 7.0.

Con la finalidad de develar si existían diferencias significativas en las escalas diaria y estacional (meses y horas) para los valores de las pendientes obtenidas para $K_{d}$, $\mathrm{TD}_{\text {temp }} \mathrm{y} \mathrm{TD}_{\mathrm{RTR}}$, los datos se sometieron a un análisis de varianza (Anova) de dos vías con el paquete Statgraphics, versión 3.0. Antes del Anova, se examinaron los supuestos requeridos para dicho análisis (normalidad, aleatoriedad y homocedasticidad).

\section{Resultados}

La temperatura de la superficie del agua presentó una leve variación entre muestreos (media $=20,8{ }^{\circ} \mathrm{C}, \mathrm{V}_{\text {mín }}=18,7^{\circ} \mathrm{C}$, $\left.\mathrm{V}_{\text {máx }}=24,2^{\circ} \mathrm{C}, \mathrm{CV}=5,5 \%, \mathrm{n}=70\right)$. Esta variable no mostró

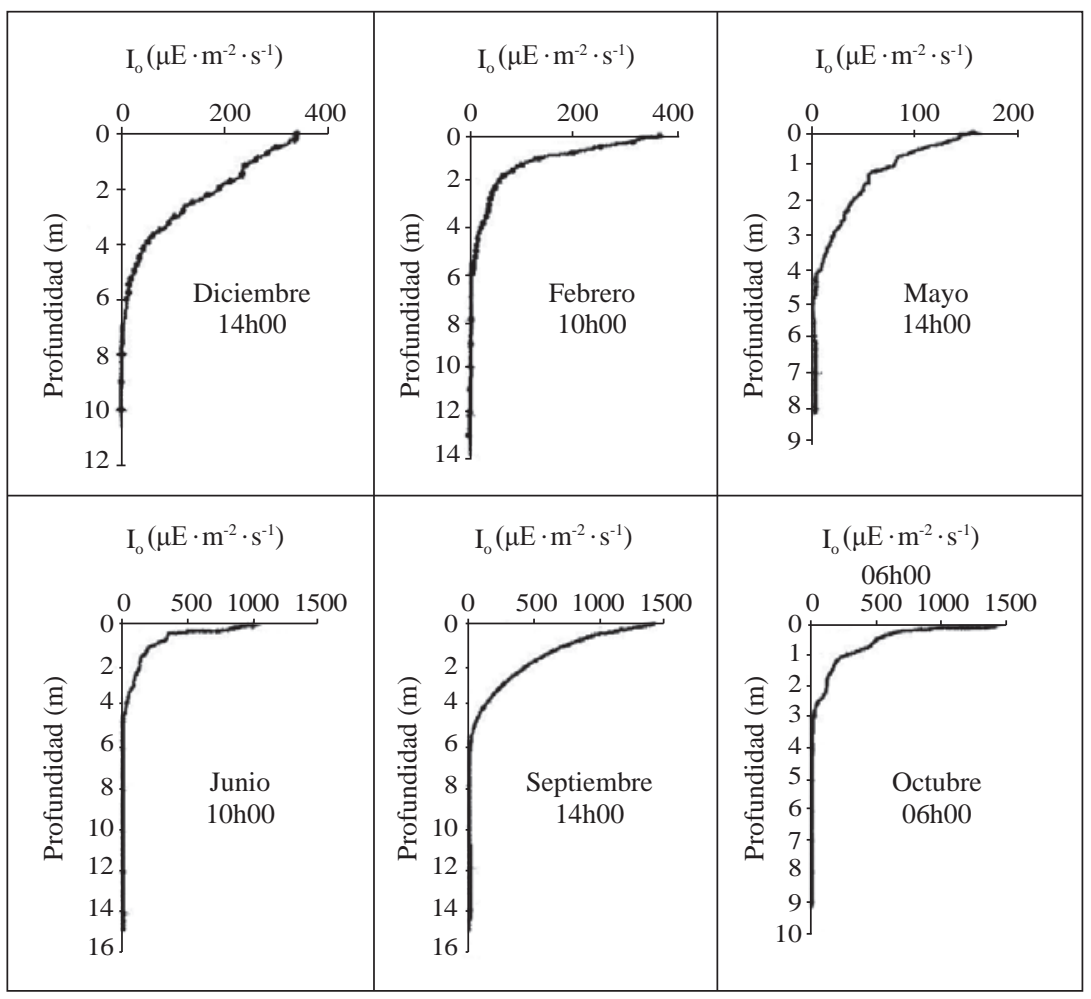

Figura 2. Embalse de La Fe. Ejemplos del comportamiento típico de los perfiles ópticos 


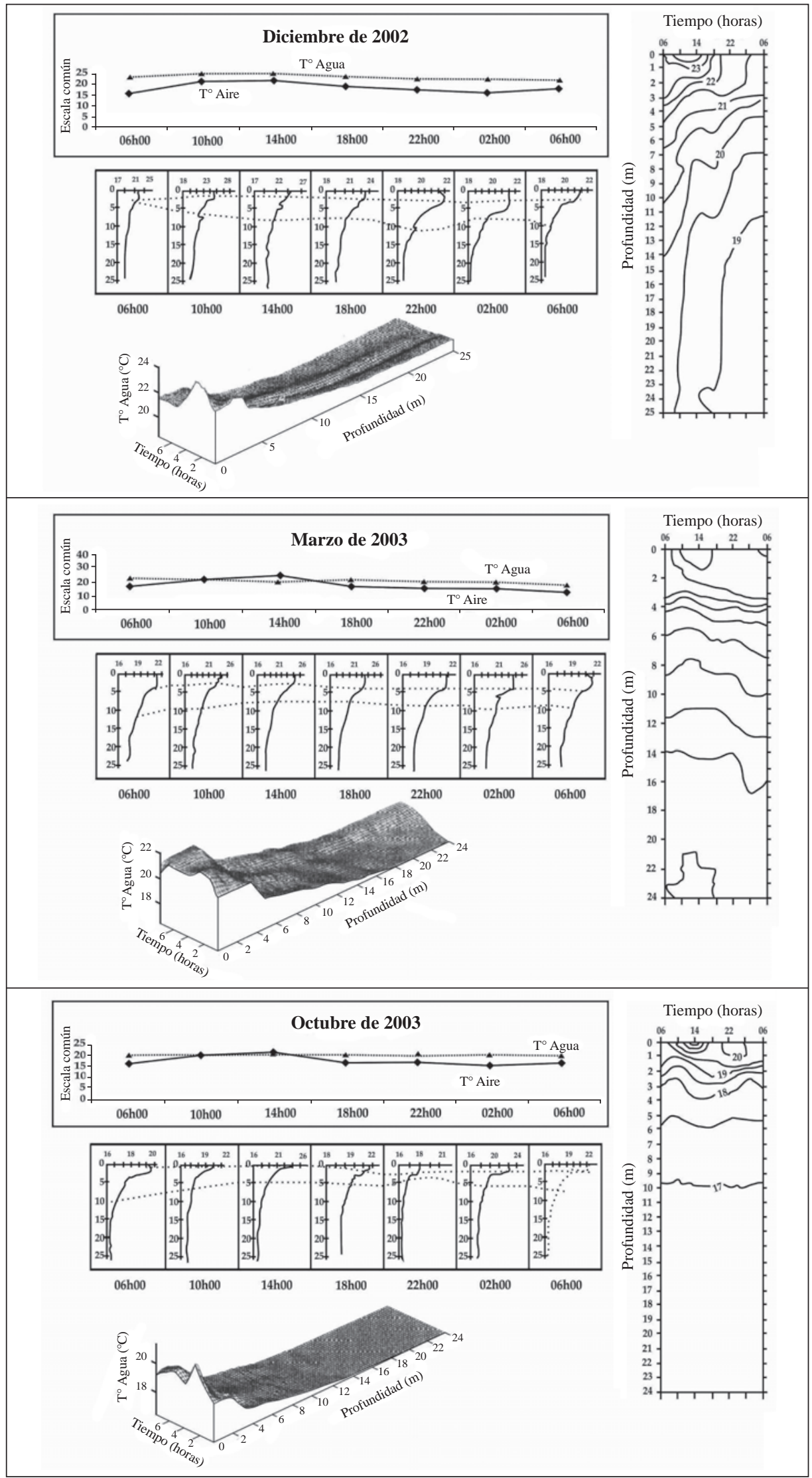

Figura 3. Embalse de La Fe. Ejemplos de los perfiles verticales diarios de temperatura 
una tendencia significativa en la escala temporal diaria ( $\alpha$ $=0,1056$ ); en la escala mensual, la tendencia fue altamente significativa y propensa a la disminución hacia los meses finales del muestreo $(\alpha=0,0000, \mathrm{r}=-0,67, \mathrm{EE}=0,85)$.

Entre horas la diferencia entre $\mathrm{T}_{\mathrm{a}}$ y $\mathrm{T}_{\mathrm{s}}$ fue altamente significativa $(\alpha=0,0001)$ y tendió a disminuir durante las horas nocturnas $(r=-0,46 ; E E=2,4)$; entre meses, dicha diferencia no fue significativa $(\alpha=0,5831)$.

La velocidad del viento en el sitio de muestreo osciló entre 1,13 (agosto) y $2,97 \mathrm{~m} / \mathrm{s}$ (junio), con un valor medio de 1,86 y una variación general no muy pronunciada $(\mathrm{CV}=$ 20,8 \%). Su dirección media predominante fue bastante uniforme ( $\mathrm{CV}=10,4 \%$ ) y se mantuvo en el sentido del eje longitudinal del lago la mayor parte del tiempo (NNOSSE), variando principalmente en diciembre de 2003 y agosto de 2004. Vale la pena anotar que el sitio de muestreo se encontraba hacia el sur.

La tendencia temporal diurna del logaritmo de la velocidad del viento fue levemente significativa $(\alpha=0,0302)$ y con una propensión muy leve a disminuir durante las horas nocturnas $(r=-0,26, \mathrm{EE}=0,77)$.

El viento no influyó significativamente en las variaciones de la temperatura del agua en la superficie $(\alpha=0,678)$, pero sí mostró relación directa con el resultado de la diferencia $\mathrm{T}_{\mathrm{a}}$ $\mathrm{T}_{\mathrm{s}}(\alpha=0,0000, \mathrm{r}=0,48, \mathrm{E} \cdot \mathrm{E}=2,3)$.

El coeficiente de atenuación vertical para irradiancia descendente $\left[\mathrm{K}_{\mathrm{d}}(\mathrm{PAR})\right]$, utilizado para evaluar la estructura óptica, mostró una media de $0,19 \mathrm{~m}^{-1}$, un rango de $0,23 \mathrm{~m}^{-1}$ $\mathrm{y}$ una leve tendencia significativa tanto en la escala diaria $(\alpha=0,0312)$ como en la anual $(\alpha=0,0459)$. El inverso de la transparencia (horas: $\alpha=0,5923$, meses: $\alpha=0,2704$ ), no arrojó tendencias significativas en ninguna de las dos escalas temporales investigadas. Igual sucedió con el primer criterio de evaluación de la forma térmica ( $\mathrm{TD}_{\text {temp }}$ ) (horas: $\alpha=0,2980$; meses de muestreo: $\alpha=0,6425$ ), el cual osciló entre 0,5 y $7,6{ }^{\circ} \mathrm{C} / \mathrm{m}$ para un valor medio de $4,2{ }^{\circ} \mathrm{C} / \mathrm{m}$ ). El segundo criterio de evaluación de la forma térmica $\left(\mathrm{TD}_{\mathrm{RTR}}\right)$ presentó una leve tendencia significativa en la variación temporal diaria $(\alpha=0,0359)$, mas no en la estacional ( $\alpha=$ 0.1986), oscilando entre - 0,02 $\mathrm{m}^{-1} \mathrm{y}-0,25 \mathrm{~m}^{-1}$ (media: - 0,29 $\left.\mathrm{m}^{-1}\right)$. El promedio de la transparencia fue de $2,7 \mathrm{~m}$ y su rango de 2,1 m; su variabilidad en la escalas mensual y estacional no fue significativa (Tablas 2, 3 y 4 ).

La figura 2 presenta algunos ejemplos de variación de la estructura óptica del embalse; puede observarse que la luz penetró hasta una profundidad que osciló entre los 4 y 6 metros, lo que implica una zona iluminada equivalente a un porcentaje entre el 16 y el $24 \%$ de la columna de agua en la presa del embalse.

Algunos ejemplos significativos de la forma general de la curva de estratificación térmica (Figura 3) muestran que fue bastante similar a una curva exponencial monotónica
Tabla 2. Significancia estadística $(\alpha)$ de la variación espacial y temporal de las tasas de cambio de la temperatura con la profundidad, la transparencia (Dsd), la estabilidad y el coeficiente de atenuación vertical para irradiancia descendente $\left(\mathrm{K}_{\mathrm{d}}(\mathrm{PAR})\right]$.

\begin{tabular}{llc}
\hline Variable dependiente & Factor & $\boldsymbol{\alpha}$ \\
\hline $\begin{array}{l}\text { Tasa de cambio de la estructura } \\
\text { óptica }\left(\mathrm{K}_{\mathrm{d}}(\mathrm{PAR}) \text { en } \mu \mathrm{E} / \mathrm{m}^{2} / \mathrm{s}\right)\end{array}$ & Horas & $0,0312^{*}$ \\
\hline 1/Dsd & Meses & $0,0459 \mathrm{~ns}$ \\
& Horas & $0,5223 \mathrm{~ns}$ \\
\hline $\begin{array}{l}\text { Tasa de cambio de la estructura } \\
\text { térmica }(\mathrm{TD}\end{array}$ temp $\left.^{\circ} \mathrm{C} / \mathrm{m}\right)$ & Meses & $0,2704 \mathrm{~ns}$ \\
\hline $\begin{array}{l}\text { Tasa de disminución de la RTR } \\
\text { (estabilidad) }\left(\mathrm{TD}_{\mathrm{RTR}}, \mathrm{m}^{-1}\right)\end{array}$ & Horas & $0,2980 \mathrm{~ns}$ \\
\hline
\end{tabular}

* Significativo con $\alpha<0,05$; ns = no significativo $(\alpha>0,05)$.

Tabla 3. Valores de la media aritmética para las tasas de cambio de la estructura óptica $\left(\mathrm{K}_{\mathrm{d}}\right) \pm$ una desviación estándar, de la estructura térmica $\left(\mathrm{TD}_{\text {temp }}\right)$ y de la estabilidad $\left(\mathrm{TD}_{\mathrm{RTR}}\right)$. El valor de n para estos cálculos es de 10, equivalente al número de muestreos nictémeros efectuados. Los valores negativos indican que la variable disminuyó hacia el fondo en cada muestreo.

\begin{tabular}{lccc}
\hline Tiempo & $\begin{array}{c}\mathbf{K}_{\mathrm{d}}(\mathbf{P A R}) \\
\left(\mathbf{m}^{-1}\right)\end{array}$ & $\begin{array}{c}\mathbf{T D}_{\text {temp }} \\
(\mathbf{O} \mathbf{C} / \mathbf{m})\end{array}$ & $\begin{array}{c}\mathbf{T D}_{\mathbf{R T R}} \\
\left(\mathbf{m}^{-1}\right)\end{array}$ \\
\hline Diciembre & $-0,19 \pm 0,04$ & $-7,6$ & $-5,1$ \\
\hline Febrero & $-0,18 \pm 0,0$ & $-2,3$ & $-5,7$ \\
\hline Marzo & $-0,17 \pm 0,06$ & $-4,4$ & $-4,1$ \\
\hline Abril & $-0,20 \pm 0,09$ & $-2,6$ & $-4,6$ \\
\hline Mayo & $-0,21 \pm 0,06$ & $-5,1$ & $-4,8$ \\
\hline Junio & $-0,22 \pm 0,04$ & $-4,2$ & $-4,6$ \\
\hline Agosto & $-0,02 \pm 0,04$ & $-4,4$ & $-3,5$ \\
\hline Septiembre & $-0,21 \pm 0,03$ & $-5,2$ & $-3,9$ \\
\hline Octubre & $-0,25 \pm 0,11$ & $-0,5$ & $-5,1$ \\
\hline Noviembre & $-0,25 \pm 0,09$ & $-5,8$ & $-0,1$ \\
\hline Media $\pm S$ & $-0,19 \pm 0,06$ & $-4,2$ & $-4,2$ \\
\hline
\end{tabular}

negativa. Generalmente, en las horas nocturnas se originó una pequeña zona de isotermia. La inclinación de la termoclina fue leve y durante todo el tiempo la mezcla estuvo restringida a la parte superficial del sitio de muestreo; por ello, la profundidad de la capa mezclada varió relativamente poco: entre $1,1 \mathrm{~m}$ en noviembre y 4,0 $\mathrm{m}$ en marzo, con un valor medio de 2,7 $\mathrm{m}$ y un CV de 36,1 \%. La tendencia general fue hacia la disminución en los últimos meses de muestreo.

La profundidad de la capa mezclada respondió en un $69 \%$ a la dirección del viento $(\alpha=0,0027, \mathrm{r}=0,83)$. La velocidad del viento solo explicó el 1,1 \% del comportamiento de dicha capa $(\alpha>0,05)$. La relación entre $\mathrm{T}_{\mathrm{a}} \mathrm{y} \mathrm{T}_{\mathrm{s}} \mathrm{y}$ la profundidad de la zona de mezcla no fue significativa $(\alpha=0,3520)$. 
Tabla 4. Valores de la media aritmética en el eje gravedad-luz para la transparencia $\left(\mathrm{D}_{\mathrm{sd}}\right)$, la conductividad eléctrica (C.E.), el pH, la tasa de cambio del oxígeno disuelto $\left(\mathrm{TDO}_{2}\right)$ y el $\mathrm{CO}_{2}$ total $\left(\mathrm{CO}_{2} \mathrm{~T}\right)$. El valor de n para estos cálculos es de 10 , equivalente al número de muestreos nictémeros efectuados, excepto para la conductividad eléctrica $(n=8)$. Los valores negativos de $\mathrm{TDO}_{2}$ indican que la variable disminuyó hacia el fondo en cada muestreo.

\begin{tabular}{|c|c|c|c|c|c|}
\hline Tiempo & Dsd (m) & C. E. $\left(\mu S . \mathrm{cm}^{-1}\right)$ & pH (Unidades de pH) & $\mathrm{TD}_{\mathrm{O} 2}\left(\mathrm{mgO}_{2}, \mathrm{I}^{-1} \mathrm{~m}^{-1}\right)$ & $\mathrm{CO}_{2} \mathrm{~T}\left(\mathrm{mgCO}_{2} \mathrm{I}^{-1}\right)$ \\
\hline Diciembre & 2,0 & 42,6 & 6,09 & $-0,19$ & 35,2 \\
\hline Febrero & 3,1 & 40,7 & 6,01 & $-0,16$ & 63,0 \\
\hline Marzo & 2,7 & 44,5 & 6,25 & $-0,16$ & 58,2 \\
\hline Abril & 2,3 & 41,9 & 6,33 & $-0,16$ & 76,2 \\
\hline Mayo & 3,2 & 41,5 & 6,37 & $-0,15$ & 37,01 \\
\hline Junio & 2,2 & 38,7 & 6,01 & $-0,19$ & 55,2 \\
\hline Agosto & 4,1 & 36,8 & 6,24 & - 0,15 & 40,9 \\
\hline Septiembre & 3,2 & 47,7 & 6,37 & $-0,15$ & 33,0 \\
\hline Octubre & 2,0 & $*$ & 6,37 & $-0,12$ & 45,4 \\
\hline Noviembre & 2,3 & $*$ & 6,33 & $-0,22$ & 40,9 \\
\hline Media & 2,7 & 41,8 & 6,2 & $-0,20$ & 48,5 \\
\hline CV (\%) & 25,1 & 8,0 & 2,4 & 16,7 & 29,1 \\
\hline
\end{tabular}

Por su parte, la variación de la temperatura del fondo (media $=17,5^{\circ} \mathrm{C}, \mathrm{V}_{\text {mín }}=16,1^{\circ} \mathrm{C}, \mathrm{V}_{\text {máx }}=19,6^{\circ} \mathrm{C}, \mathrm{CV}=5,1 \%, \mathrm{n}=$ 70) fue levemente inferior que la de la superficie. La diferencia entre $\mathrm{T}_{\mathrm{a}} \mathrm{y} \mathrm{T}_{\mathrm{s}}$ (media $=-3,5^{\circ} \mathrm{C}, \mathrm{V}_{\text {mín }}=-9,7^{\circ} \mathrm{C}, \mathrm{V}_{\text {máx }}$ $=3,7^{\circ} \mathrm{C}, \mathrm{CV}=76,3 \%, \mathrm{n}=70$ ) presentó una gran variación. La variación horaria de la temperatura del fondo tampoco presentó tendencia significativa $(\alpha=0,6097)$, pero la tendencia anual sí fue altamente significativa $(\alpha=0,0000, \mathrm{r}$ $=-0,83, \mathrm{EE}=0,49)$, $\mathrm{y}$ al igual que la $\mathrm{T}_{\mathrm{s}}$, disminuyó hacia el final del muestreo.

Los valores medios de conductividad eléctrica fueron relativamente bajos, los del pH levemente ácidos, y altos los de $\mathrm{CO}_{2}$ total en los muestreos nictémeros efectuados durante los meses del estudio. Los valores de los estadísticos de dispersión absoluta del $\mathrm{pH}$ y la tasa de cambio del oxígeno fueron también bajos; los de la transparencia, la conductividad y el $\mathrm{CO}_{2}$ total fueron en general altos (Tabla 4). Las figuras 4 y 5 ilustran el patrón general de cambio vertical para estas variables, el cual correspondió a disminuciones rápidas de oxígeno hasta la hipoxia y la anoxia completa en algunos muestreos a profundidades entre los 12 y 16 m, y a disminuciones de $\mathrm{pH}$ y aumentos de conductividad eléctrica $\mathrm{y} \mathrm{CO}_{2}$ total hacia el fondo del cuerpo de agua.

Por último, la figura 6 muestra un modelo gráfico del comportamiento general del embalse.

\section{Discusión}

\section{Estructura óptica}

Respecto a la forma óptica del embalse, podemos decir inicialmente que el valor del parámetro morfométrico Dr mencionado anteriormente predijo un embalse con transparencia alta, atenuación lumínica baja y zona fótica relativamente grande y poco variable. Según Straškaba, et al. (1993), los tiempos de retención prolongados aumentan las tasas de sedimentación y la profundidad de la zona fótica, así como la duración de los flujos superficiales, en tanto que la de los de fondo se reduce, por lo que predominan los flujos intermedios, todo lo cual se ajusta plenamente a lo encontrado en esta investigación.

El cinturón de macrófitas que rodea el embalse también es un factor que puede contribuir al incremento de la transparencia y la profundidad óptica, pues retiene los aportes de sedimentos que entran por los afluentes. Además, se considera que en los embalses la zona pelágica generalmente, presenta mayor tiempo de residencia que las demás zonas, por lo que se espera un incremento de la tasa de sedimentación del material particulado y, consecuentemente, de la transparencia. La investigación de Vargas \& Ramírez (2002) demostró que no había diferencia entre las tasas de sedimentación de las zonas media y de presa en este embalse, lo que permite pensar que el embalse de La Fe posee una gran zona limnética que se extiende hasta la zona media.

Dado que los organismos de la comunidad de fitoplanctón registrados en otras investigaciones son bastante grandes (Botryococcus braunii, Staurastrum spp, Microcysitis aeruginosa, Woronochinia naegeliana, Peridinium c.f. gatunense, Ceratium furcoides), el sombreo proporcionado por las algas no se considera un factor que afecte plausiblemente la penetración de luz.

Ramírez, et al., (2001) plantean que a medida que se incrementa la altura a la que se encuentra un cuerpo de agua, las condiciones edáficas varían y ocurre un menor arrastre de sedimentos y otros materiales; se espera, entonces, que en los lagos y embalses situados a mayor altura y con 


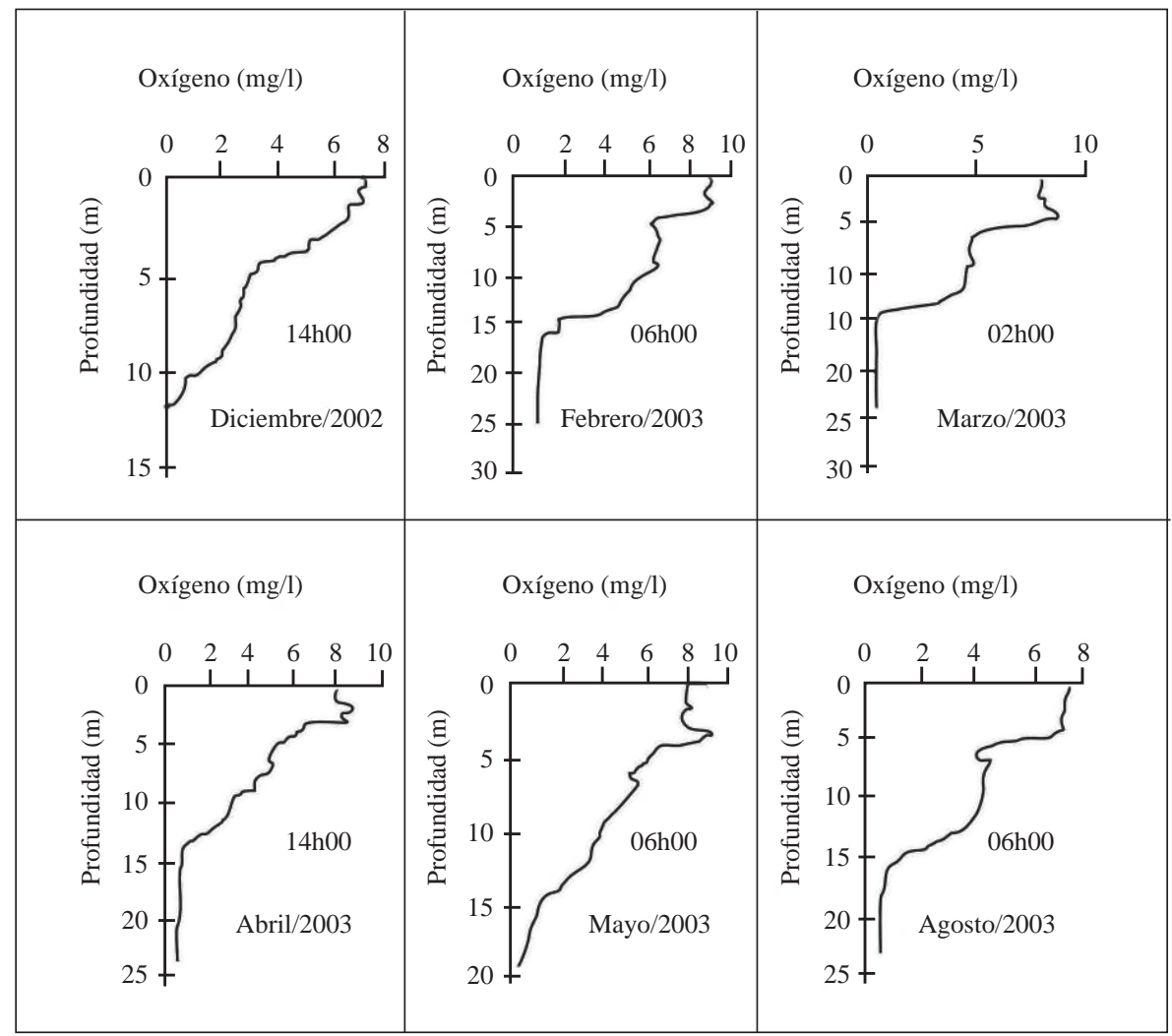

Figura 4. Embalse de La Fe. Ejemplos de la conducta del oxígeno en el eje gravedad-luz

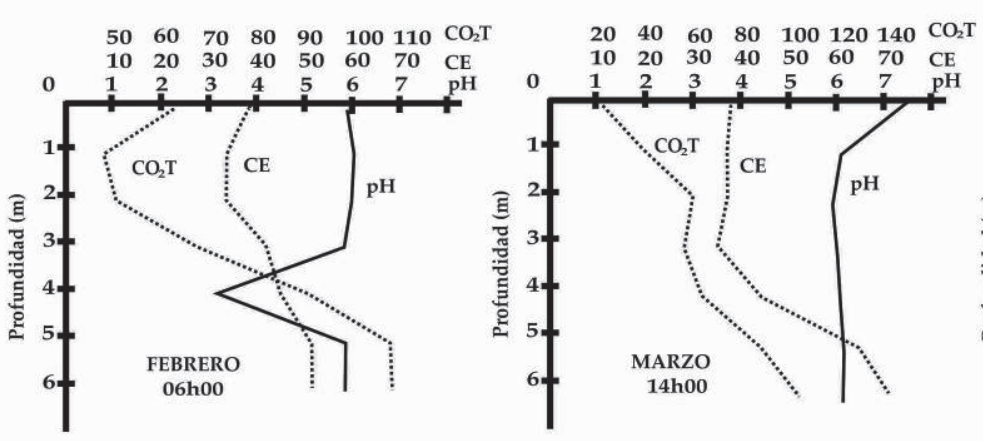

$\begin{array}{llllllllllll}30 & 60 & 90 & 120 & 150 & 180 & 210 & 240 & \mathrm{CO}_{2} \mathrm{~T}\end{array}$
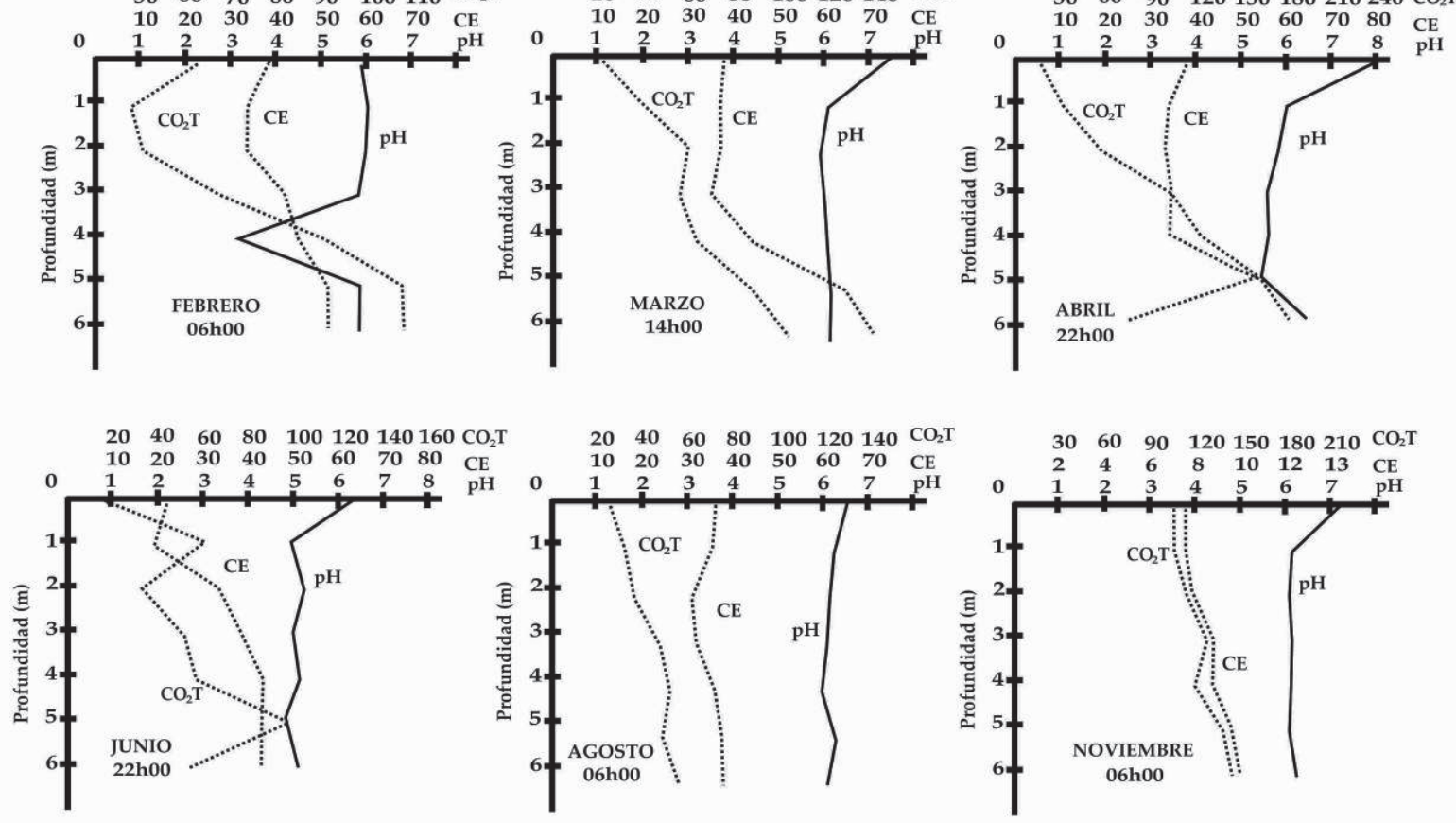

Figura 5. Embalse de La Fe. Ejemplos del comportamiento general de los perfiles de conductividad eléctrica $(\mathrm{C} . \mathrm{E}$.$) , \mathrm{pH}$ y $\mathrm{CO}_{2}$ total 


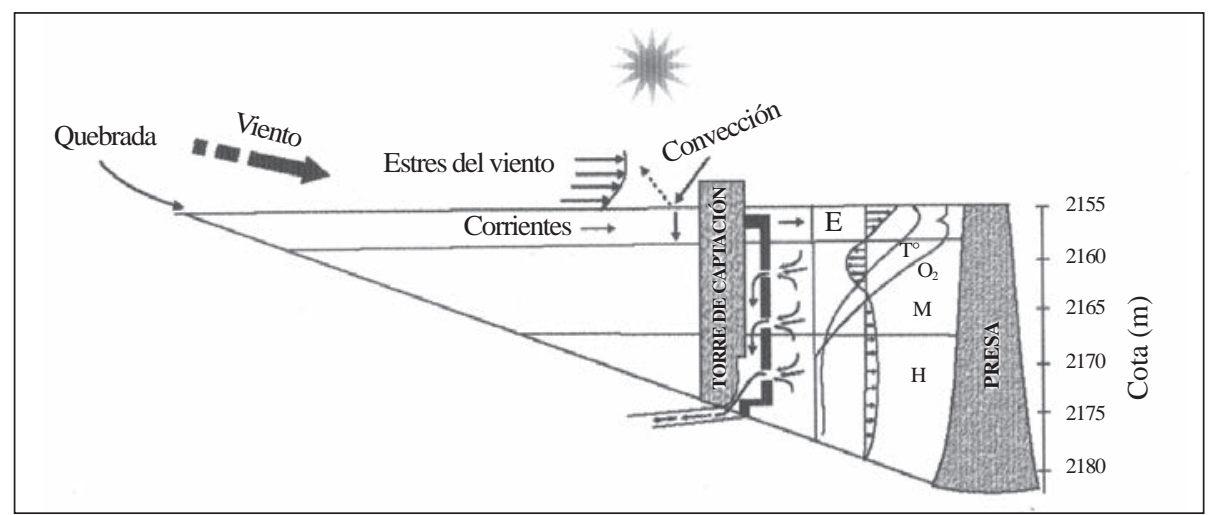

Figura 6. Modelo gráfico general del comportamiento de la columna de agua en el embalse

buena protección vegetal, la transparencia y la penetración de luz sean mayores. El embalse de La Fe se ubica a una altura de $2.100 \mathrm{~m}$, con una protección vegetal adecuada a su alrededor. Al igual que los embalses de San Lorenzo y El Peñol, se encuentra en la zona conocida como "batolito antioqueño”, la cual, según Espinal (1985), se deriva de cenizas volcánicas y rocas ígneas que ceden poco material por lixiviación, a pesar de que la pluviosidad de la zona sea alta (entre 2.000 y $4.000 \mathrm{~mm}$ /año para la zona de vida del embalse) y se presenten pendientes considerables.

Debe considerarse, además, que el tratamiento previo de las aguas del río Pantanillo en la planta localizada en el municipio de El Retiro antes de ser bombeadas a la represa de La Fe, disminuye considerablemente el aporte de material particulado que pueda interferir en la penetración de luz en la presa del embalse (R amírez, 1999).

Los datos referentes a la profundidad fótica muestran que la luz en este cuerpo de agua penetra mucho más allá de la profundidad límite del epilimnio. Lo anterior tiene, al menos en teoría, profundas implicaciones para el fitoplancton, dado que cuando la zona de mezcla es inferior a la zona fótica, el fitoplancton está sometido a niveles altos de irradiaciones medias de luz y a una alta densidad del flujo de fotones en escalas temporales cortas (minutos, horas y días), y no experimenta en estas escalas ni saturaciones de luz por debajo de los niveles normales, ni periodos de oscuridad, ni alternancias día/noche, ni modificaciones sustanciales en el ambiente subacuático de la fotosíntesis dentro de cada ciclo de mezcla. Sin embargo, esta situación, aparentemente favorable, podría llegar a superar la capacidad homeostática de las células algales frente a la variabilidad ambiental de corto plazo, afectando la producción primaria neta al incrementarse las tasas respiratorias.

Aunque existió una baja variabilidad de la forma óptica (desviación estándar entre 0,0 y 0,11) en la escala diaria, esta fue levemente significativa $(\mathrm{a}=0,0312)$, lo cual indica que es relativamente inestable en la escala diaria mas no en las escalas mensual y anual; por ello, los perfiles verticales de atenuación que se muestran en la figura 2 son muy similares entre meses y a lo largo del año, como se espera que ocurra en la zona tropical (Ganf \& Horne, 1975; Schiemer \& Boland, 1996; M argalef, 1983) (Tabla 3).

\section{Clasificación óptica}

Desde el punto de vista óptico, las aguas del embalse pueden clasificarse inicialmente como pertenecientes al tipo GA en el sistema de K irk (2011), en las cuales el gilvin absorbe más que la fracción particulada y la turbidez es relativamente baja (turbidez media: 10,6 NTU, n = 284, en esta investigación). Las razones que permiten colegir lo anterior se fundan en que en La Fe: 1 ) el color (media: 51,6; $n=284$, en esta investigación) es relativamente alto e indicador de aguas con una contribución sustancial de substancias amarillas (material orgánico disuelto coloreado [colored dissolved organic matter - CDOM] o gilvin), que se originan cuando el tejido vegetal se descompone en el suelo del área de captación o en el agua; 2) el cinturón litoral de E. densa ya mencionado, que conjuntamente con las floraciones de diversos organismos de fitoplancton como Botryococcus braunii, Staurastrum spp., Woronochinia naegeliana, Cuspidothrix sp. y Ceratium furcoides, conforman aportes autóctonos de material orgánico; 3) el área de captación, con fuentes de material vegetal alóctono en descomposición provenientes de los cultivos de flores, potreros y plantaciones forestales de pino y ciprés, y 4) los afluentes, que contribuyen también con materia orgánica alóctona proveniente de las descargas de aguas residuales domésticas.

\section{Estructura térmica}

En los lagos de regiones templadas, la zona de mezcla presenta un espesor bastante constante, perturbado únicamente por el avance de la estación fría, que disminuye la temperatura y aumenta la densidad de las capas superiores, produciendo como resultado la erosión de la termoclina y el consiguiente aumento de la zona de mezcla. Con respecto a los lagos tropicales y ecuatoriales se ha dicho que este mecanismo no es tan claro, y que el espesor de la zona de mezcla es muy variable (B eadle, 1974; L ewis, 1987); en concordancia con 
esta última proposición, el grueso de dicha zona presentó efectivamente una variación relativamente alta $(\mathrm{CV}=36,1 \%)$, que no se explica por la variación en la velocidad del viento ni por las fuerzas convectivas (diferencia $T_{a}-T_{s}$ ), pero sí por la dirección del viento (en un 69 \%).

Según varios autores (Talling, 1963; Beadle, 1974; Cole, 1983; L ewis, 1983a; L ehmusluoto, et al., 1999; Umaña, et al., 1999; León-López, et al., 2012), los lagos ecuatoriales y tropicales presentan pequeñas diferencias verticales de temperatura, suficientes, sin embargo, para generar una estratificación estable. Álvarez-C obelas (1992) propone que un gradiente térmico de $0,3{ }^{\circ} \mathrm{C}$ es suficiente para el desarrollo de una estructura térmica estable.

En general, la profundidad de la mezcla del epilimnio está en función de la velocidad y la frecuencia media del viento y del fetch. Se considera que un estrés de viento con una velocidad mayor de $3,5 \mathrm{~m} / \mathrm{s}$ produce un flujo de energía cinética que genera energía cinética turbulenta, la cual distribuye el flujo neto de calor a través de la capa mezclada hasta cuando disminuye la velocidad y alcanza el equilibrio. Salvo en el mes de junio, la velocidad del viento no alcanzó dichos valores en la presa del embalse de La $\mathrm{Fe}$, por lo que estas fuerzas no fueron lo suficientemente eficaces para mezclar el agua y erosionar la termoclina, la cual se localizó muy cerca a la superficie. Por lo tanto, no hay un epilimnio claramente definido y el gradiente térmico comienza directamente en la superficie.

Como consecuencia de lo anterior, el patrón de forma térmica resultante se caracterizó por no variar significativamente ni en la escala diaria ni en la mensual, pero sí por presentar periodos de mezcla irregulares e incompletos, poco variables y causados, sobre todo, por las corrientes de convección que se repiten a diario, las lluvias fuertes, o los afluentes. R omán, et al., (2013) plantean que el aumento del caudal natural de los afluentes durante las épocas de lluvia y la ausencia del bombeo en estos periodos disminuyen el gradiente térmico debido a la incursión de dichos afluentes hacia el cuenco sur, y a que, probablemente, el agua del fondo es reemplazada por las aguas frías producto de las crecientes, favoreciendo así la mezcla vertical. La pluma de agua originada en el bombeo es para estos autores la responsable de esos cambios verticales en la columna de agua.

Por ello, y en concordancia con lo planteado por $\mathbf{H}$ utchinson \& Löffler (1956), Lewis (1973, 1983b) y Tundisi \& Matsumura-Tundisi (2008), en el embalse de La Fe la mezcla es incompleta y los periodos de circulación tienden a ser inusuales, irregulares y de corta duración. Datos provenientes de lagos tropicales muestran que la mezcla causada únicamente por la convección es probablemente superficial (L ewis, 1987). L ewis (1973) denominó a este patrón atelomixis $($ atelos $=$ incompleto, mixis $=$ mezcla $)$, y lo describió como el calentamiento térmico diurno y el enfriamiento nocturno, lo cual produce variaciones diurnas de temperatura que pueden exceder las estacionales (anuales). Tales variaciones térmicas y de densidad ocurren en toda la columna de agua, no solamente en el epilimnio, cuando las estratificaciones son estables con termoclinas primarias bien establecidas. En ese caso, la atelomixis se denomina parcial y puede considerarse un fenómeno común en lagos tropicales (Talling \& Lemoalle, 1998; Barbosa \& Padisàk, 2002). Este es un fenómeno de importancia fundamental en la reorganización vertical de la estructura térmica y la densidad de los lagos y sus interacciones con la distribución vertical del fitoplancton y los nutrientes (Tundisi \& M atsumura-Tundisi, 2008).

Ruttner (1953) plantea que de 15 lagos indonesios estudiados por él y Thienemann en 1830, la mayoría estaba estratificado y su estabilidad se relacionaba con el área y la profundidad: lagos con un área menor de $2 \mathrm{~km}^{2}$ eran más estables térmicamente, aquellos con un área aproximada de $100 \mathrm{~km}^{2}$ mostraban menor estabilidad; los lagos con un área de más de $1.000 \mathrm{~km}^{2}$ casi no presentaban estabilidad. Dado que el embalse de La Fe tiene un área de $1,43 \mathrm{~km}^{2}$, se ajustaría al primer tipo de lagos considerados por $\mathbf{R}$ uttner, es decir, un lago térmicamente estable.

K ling (1988) define la profundidad de mezcla como la razón entre la profundidad máxima y el área, lo que en el embalse de La Fe da un resultado de 1,96 m y permite prever que en cualquier sitio del embalse la profundidad del epilimnio no sea considerable. El valor medio hallado para la zona de mezcla fue de $2,7 \mathrm{~m}$ (10,8 \% de la columna total de $25 \mathrm{~m})$, el cual, en términos generales, se ajusta a la previsión. Además, los perfiles térmicos establecidos en seis estaciones de muestreo durante el mismo periodo de este estudio, indican que efectivamente el patrón incompleto de la atelomixis se presenta en todo el embalse.

Otro predictor teórico de la posibilidad de mezcla de un cuerpo de agua es la profundidad relativa (Dr). Según este parámetro morfométrico, los cuerpos de agua con un área superficial grande y poca profundidad tienen tendencia a mezclarse, mientras que los lagos y embalses pequeños y profundos tienen valores altos de Dr y se mezclan poco. El valor de este parámetro morfométrico en el embalse es de $2,07 \%$, que se considera relativamente alto, lo que significa que solo un 2,1\% de la profundidad del cuerpo de agua se presenta mezclado, bien sea por fuerzas convectivas o mecánicas (Hernani \& Ramírez, 2002). Aunque el valor medio de la zona de mezcla no corresponde al $2 \%$ predicho, en términos generales sí muestra la poca mezcla presente.

Como ya se mencionó, la dirección dominante del viento fue hacia la zona de presa, localizada hacia el SSE. No obstante, debido a que la estación de muestreo se sitúa en un área donde la influencia del viento queda confinada a ella, no alcanza a desarrollar el trabajo suficiente para vencer las fuerzas viscosas que se oponen a la mezcla profunda. 
Otro factor que dificulta la mezcla total de la columna de agua es la protección que ejercen la vegetación, las montañas y las construcciones alrededor de la represa, que evitan la acción directa del viento. Los valores medios de la fuerza eólica oscilaron entre brisa leve y vientos suaves en la escala de Beaufort, influencia ésta muy pequeña para vencer las fuerzas viscosas. La circulación y la mezcla quedan así restringidas al epilimnio, tal como ocurre en otros embalses tropicales (Tundisi, et al., 1981; Tundisi \& MatsumuraTundisi, 1984; Infante \& Infante, 1994; Soto \& L ópez, 1996; González, et al., 2002; Tundisi \& MatsumuraTundisi, 2008).

Como puede verse, existen suficientes evidencias para rechazar la hipótesis de Villamizar (1984) de que este cuerpo de agua, una vez en funcionamiento, no presentaría estratificación térmica estable.

La influencia significativa de la diferencia entre $T_{s}$ y $T_{a}$ se debe, según L ewis $(1987,1996)$, a la mayor pérdida nocturna de calor en latitudes bajas, ya que cuando la temperatura es alta una determinada pérdida de calor desde el agua ocasiona mayores cambios de densidad. Para Imberger (1985), una interacción entre aire y agua inestable puede causar un intercambio de hasta el $100 \%$ en la transferencia de calor y masa durante episodios de viento leve $(<2 \mathrm{~m} / \mathrm{s})$, cuando predomina la mezcla convectiva en la capa fronteriza.

G onzález, et al., (2004), Straškaba, et al. (1999) y Henry (1999) plantean que los embalses con tiempos de residencia mayores a 200 días pueden desarrollar estratificación térmica estable. Sin embargo, el embalse de La Fe, con un tiempo de residencia mucho menor, de aproximadamente 28 días, desarrolla — según se ha descrito en parágrafos anterioresuna estratificación estable.

Por presentar perfiles térmicos heterogéneos con diferencias de $5^{\circ}$ y $6{ }^{\circ} \mathrm{C}$ entre superficie y fondo (por lo que pueden esperarse planos horizontales de densidad constante durante periodos de vientos débiles y de disminución en las entradas o salidas), así como un hipolimnio anóxico, aumentos de $\mathrm{CO}_{2}$ total y conductividad eléctrica hacia el fondo y disminuciones de $\mathrm{pH}$ en esta misma profundidad, el embalse de La Fe se ajusta a lo que Tundisi \& M atsumura-Tundisi (1984; 2008) denominan embalses con estratificación permanente. Según estos autores, estos sistemas ocurren en ríos con valles profundos y cuando la salida de agua para las turbinas se encuentra a profundidades intermedias entre la superficie y el fondo de la represa (Figura 6).

La anoxia de fondo hallada se debió fundamentalmente al patrón térmico encontrado, que aísla el hipolimnio de la inyección de oxígeno ocasionada por la mezcla en una columna holomíctica. Además, un embalse con un período de retención hidráulica largo tiene una mayor probabilidad de presentar una estratificación que lo lleve a un estado de anoxia permanente en el fondo que uno con un tiempo de retención menor. Fuera de eso, las temperaturas de fondo de los embalses y lagos tropicales y ecuatoriales son altas (> $20{ }^{\circ} \mathrm{C}$ en promedio), en comparación con los de zona templada ( $15{ }^{\circ} \mathrm{C}$ en promedio, aproximadamente); por lo tanto, en concordancia con la regla de Vant' H off, y debido al consumo de oxígeno 4 a 9 veces más rápido, estos cuerpos de agua presentan una tendencia normal a la anoxia de fondo. Como consecuencia del mayor consumo de oxígeno, el $\mathrm{CO}_{2}$ y otros solutos se liberan más rápidamente y la materia orgánica sedimentada se mineraliza a mayor velocidad (R uttner, 1953; E steves, 1998; Schiemer \& Boland, 1996; Lewis, 2000 Tundisi \& M atsumura-Tundisi, 2008).

Las altas temperaturas de la superficie $\left(>28^{\circ} \mathrm{C}\right)$ son un rasgo tropical permanente de muchos lagos y embalses suramericanos, africanos y asiáticos (Talling, 1965; Beadle, 1974; E ccles, 1974; Beeton, 1991; F rempong, 1995; R oldán \& R uiz, 2001). Debido a las altas temperaturas de estas aguas, la descomposición progresa rápidamente, de tal forma que en un período corto pueden tener lugar profundos cambios químicos en el hipolimnio, haciéndolo inhabitable para peces y otros organismos aeróbicos.

Según Thornton (1990), los embalses con valores bajos de fetch, profundidad moderada (aproximadamente $20 \mathrm{~m}$ ), estratificación leve a fuerte y operados para liberación de aguas cálidas pueden desarrollar una zona anóxica cerca de la presa, la cual puede extenderse verticalmente en la columna de agua y también en sentido horizontal en las demás zonas. Los resultados registrados hasta ahora muestran que estas condiciones se cumplen en el embalse de La Fe y conforman una explicación alternativa para la anoxia hipolimnética que se presenta.

Así pues, el sistema del embalse de La Fe se mantiene estacionario y a un nivel bajo de desorden (poca entropía), lo que se explica por la disipación del calor desde la superficie y a su poca distribución en el sistema debido a la ausencia de eventos de mezcla profundos producto del patrón atelomíctico. De ahí las diferencias no significativas de la tasa de cambio de la temperatura en las escalas diaria (a $=0,2980)$ y mensual $(a=0,6425)$ y levemente significativas de la $\mathrm{TD}_{\mathrm{RTR}}$ en la escala diaria $(\mathrm{a}=0,0359)$ (Tabla 3 ).

Dado que desde las dos compuertas superiores se libera agua en forma regular y continua en un flujo lento, la mayoría del agua que pasa por ellas proviene del metalimnio, por lo que en cierta medida se incrementa el tiempo de residencia del agua hipolimnética y con ello la anoxia en esta zona. Para R omán, et al., (2013), es probable que las zonas profundas en el cuenco sur se mantengan muy quietas en temporadas de estiaje y permanezcan allí por largos periodos. Además, según Cole \& Hannan (1990), Straškaba, et al., (1999) y Tundisi \& M atsumura-Tundisi (2008), los embalses con salidas superficiales, y en algunos casos aquellos con salida intermedia, incrementan el tiempo de residencia de las aguas hipolimnéticas, lo que aumenta los procesos oxidativos causando una mayor desoxigenación en esta zona (Figura 6). 


\section{Conclusiones}

Con respecto a la estructura óptica, puede concluirse que la previsión formulada se aceptó parcialmente, pues dicha estructura varió significativamente en la escala diaria pero no en la estacional. Por sus características, el embalse de $\mathrm{La} \mathrm{Fe}$ puede pertenecer ópticamente al tipo GA de K irk. Futuros estudios que profundicen más en este tema permitirán dilucidar con mayor claridad el tipo de forma óptica al que se ajusta este cuerpo de agua.

La previsión de la investigación en cuanto a la forma térmica no se aceptó, a pesar de las leves diferencias significativas de la tasa de cambio de la estabilidad en la escala diaria; por lo tanto, la pregunta inicial acerca de cómo es la variación temporal diaria y anual del patrón de forma térmico se puede responder aseverando que en el sitio de muestreo dicho patrón no mostró cambios significativos en las dos escalas temporales evaluadas (diaria y estacional).

El patrón de mezcla hallado corresponde a una atelomixis parcial, con un epilimnio poco definido. Debido al prolongado aislamiento de las capas inferiores por las densas capas superiores, el cuerpo de agua presentó un hipolimnio anóxico y bastante extenso (aproximadamente entre el 80 y el $98 \%$ de la columna de agua de 25 metros en la presa) durante todo el tiempo del muestreo, lo que permite pensar que ni los afluentes ni el bombeo de agua desempeñan un papel importante en la reaireación del embalse ni en su mezcla. Las temperaturas hipolimnéticas permanecieron relativamente constantes, los eventos de mezcla se limitaron a los primeros metros y fueron mayores en el periodo nocturno, el pH disminuyó hacia el fondo, y la conductividad y el $\mathrm{CO}_{2}$ total se incrementaron hacia esta profundidad.

\section{Agradecimientos}

El autor agradece al Comité de Investigaciones (CODI) de la Universidad de Antioquia por la financiación del proyecto inicial de "Caracterización limnológica del embalse La Fe, Antioquia, Colombia”, así como a los operarios del embalse y al ingeniero Darlin Tobón.

\section{Conflicto de intereses}

El autor declara no tener ningún conflicto de interés.

\section{Bibliografía}

Álvarez-C obelas, M. (1992). Temperature and heat in a hypertrophic, gravel pit lake. Arch. Hydrobiol. 125: 279-294.

Barbosa, F.A.R \& Padisàk, J . (1992). The forgotten lake stratification pattern: Atelomixis, and its ecological importance. Verhandlungen Internationale Vereinigung für Theoretische und Angewandte Limnologie. 28: 1385-1395.

Beadle, L.C. (1974). The inland waters of tropical Africa. An introduction to tropical limnology. London: Longman.

Beeton, A.M . (1991). Limnology of the Nam Ngum reservoir, Laos. Verhandlungen Internationale Vereinigung für Theoretische und Angewandte Limnologie. 24: 1436-1444.
Cole, G .A. (1983). Textbook of Limnology. (3ra Ed). Saint Louis: The Mosby Co.

Cole, T.M . \& Hannan, H.H . (1990). Dissolved oxygen dynamics. En K. W. Thornton, Kimmel, L. \& Payne, F.E. (Eds.). Reservoir Limnology: Ecological perspectives. New York: Wiley. p. 71-107.

E ccles, D.H . (1974). An outline of the physical limnology of Lake Malawi (Lake Nyasa). Limnology and Oceanography. 19: 730-742.

E steves, F.A . (1998). Fundamentos de limnología. Río de Janeiro: Interciencia/Finep.

Fernández, J . (2006). Modelamiento de la Estructura Térmica en el Sistema Hidroeléctrico de los Embalses Porce II - Porce III (Tesis). Facultad de Minas. Universidad Nacional de Colombia. Medellín.

Franco-Velásquez, J.D. (2011). Modelación de la estructura térmica de un embalse ramificado mediante el análisis de los procesos físicos gobernantes. Aplicación al embalse multipropósito Riogrande II (Tesis de maestría). Facultad de Minas. Universidad Nacional de Colombia. Medellín.

Frempong, E. (1995). Limnological research and training in Ghana: The past, present and perspectives for the future development. En B. Gopal y Wetzel, R.G. (Eds.). Limnology in Developing Countries, 1, New Delhi: International Association for Limnology (SIL). p. 1-39.

G anf, G.G . \& Horne, A.J . (1975). Diurnal stratification, photosynthesis and nitrogen fixation in a shallow equatorial lake (Lake George, Uganda). Freshwat. Biol. 5: 13-19.

G onzález, E J ., O rtaz, M., Peña Herrera, Carrillo, V. (2002). Caracterización limnológica de un embalse en fase de llenado, embalse Taguaza, Estado Miranda. Hidroimpacto C.A. Hidrocapital y Universidad Central de Venezuela, Caracas.

G onzález, E J ., Ortaz, M ., Peña Herrera, C ., Infante, A . (2004). Physical and chemical features of a tropical hypertrophic reservoir permanently stratified. Hydrobiologia. 522: 301310.

Henry, R . (1999). Heat budgets, thermal structure and dissolved oxygen in Brazilian reservoirs. En J. G. Tundisi. \& Straskraba, M. (Eds.). Theoretical Reservoir Ecology and Its applications. São Paulo: International Institute of Ecology, Brazilian Academy of Sciences \& Backhuys Publishers. p. 125-151.

Hernani, A \& \& Ramírez, J J . (2002). Aspectos morfométricos y teóricos de un embalse tropical de alta montaña: represa La Fe, El Retiro, Colombia. Revista de la Academia Colombiana de Ciencias Exactas Físicas y Naturales. 26: 511-518.

H utchinson, G .E . (1957). A treatise on Limnology. I. Geography, physics, and chemistry. New York: Wiley.

Hutchinson, G.E. \& Löeffler, H. (1956). The thermal classification of lakes. Proceedings of Natural Academy of Sciences, USA. 42: 84-86.

K irk, J .T.O. (2011). Light and Photosynthesis in Aquatic Ecosystems. $3^{\text {rd }}$ Ed. Cambridge: Cambridge University Press. 
Kling, G.W. (1988). Comparative transparency, depth of mixing, and stability of stratification in lakes of Cameroon, West Africa. Limnol. Oceanogr. 33: 27-40.

Imberger, J . (1985). The diurnal mixed layer. Limnol. Oceanogr. 30: $737-770$.

Infante, A. \& Infante, 0. (1994). Limnological studies in a tropical man-made lake (Lagartijo reservoir) Venezuela. Internat. Rev. Ges. Hydrobiol. 79: 17-25.

Lehmusluoto, P., M achbub, B., Terangna, N., Achmad, F., Boer, L., Brahmana, S.S., et al. (1999). Limnology in Indonesia: From the legacy of the past to the prospects for the future. En: R. G. Wetzel \& B. Gopal (Eds). Limnology in Developing Countries, 2. New Delhi: International Association for Limnology (SIL). p. 119-234.

Lewis, W.M. J r. (1973). The thermal regime of Lake Lanao (Philippines) and its theoretical implications for tropical lakes. Limnol. Oceanogr. 18: 200-217.

L ewis, W.M . J r. (1983a). Temperature, heat, and mixing in Lake Valencia, Venezuela. Limnol. Oceanogr. 28: 273-286.

L ewis, W.M . J r. (1983b). A revised classification of lakes based on mixing. Can. J. Fish. Aq. Sc. 40: 1779-1787.

L ewis, W.M . J r. (1987). Tropical limnology. An. Rev. Ecol. Syst. 18: $159-184$.

Lewis, W.M. J r. (1996). Tropical lakes: How latitude makes a difference. En: F. Schiemer. \& Boland, T. (Eds.) Perspectives in tropical limnology. Amsterdam: SBP Academic Publishing. p. 43-64.

L ewis, W.M.J r. (2000). Basis for the protection and management of tropical lakes. Lakes and Reservoir Research Management. 5: 35-48.

L eón-L ópez, N., R ivera-R ondón, C .A ., Zapata, A ., J iménez, J ., Villamil, W. A renas, G., et al. (2012). Factors controlling phytoplankton in tropical high-mountain drinking-water reservoirs. Limnetica. 31: 305-322.

M ackereth, J J ., H eron, H., Talling, J .F. (1978). Water analysis: Some revised methods for limnologists. Freshwater Biological Association, Scientific Publication n ${ }^{\circ} 36$. England: Titus Wilson \& Son Ltd.

M argalef, R . (1974). Ecología. Barcelona: Ediciones Omega, S.A.

Margalef, R . (1980). La biósfera entre la termodinámica y el juego. Barcelona: Ediciones Omega, S.A.

M argalef, R . (1983). Limnología. Barcelona: Ediciones Omega, S.A.

M argalef, R . (1993). Teoría de los sistemas ecológicos. $2^{\mathrm{a}}$ ed. Barcelona: Universitat de Barcelona Publicacions.

M arín, L . (2008). Modelamiento de la estructura térmica en embalses tropicales, aplicación Riogrande II (Tesis). Facultad de Minas. Universidad Nacional de Colombia. Medellín.

M ontoya-M oreno, Y . (2008). Variaciones nictemerales de algunas variables ambientales y fisicoquímicas en una laguna somera en Guatapé, Antioquia (Colombia). Actual. Biol. 30: 83-96.MMM
M ontoya-M oreno, Y. \& A guirre, N. (2009). Cambios nictemerales de variables físicas y químicas en la ciénaga de Paticos, complejo cenagoso de Ayapel, Colombia. Rev. Biol. Trop. 57: 635-646.

Padisák, J ., Soróczki-Pintér, E ., R eceñir, Z.S. (2003). Sinking properties of some phytoplankton shapes and the relation of form resistance to morphological diversity of plankton: An experimental study. Hydrobiologia. 500: 243-257.

Ramírez J.J. \& Díaz, A. (1995a). Variaciones verticales de temperatura y factores químicos en un ciclo de 24 horas en el embalse Punchiná, Antioquia, Colombia. Acta Limnol. Bras. 7: 23-34.

Ramírez, J J . \& Díaz,A . (1995b). Cambios diurnos de temperatura y variables físicas y químicas en dos épocas del año en la laguna del Parque Norte, Colombia. Acta Limnol. Bras. 7: 87-104.

Ramírez, J J ., Bicudo, C.E.M., Roldán, P.G., García, L.C. (2001). Variación vertical de parámetros físicos y químicos en cuatro embalses tropicales y su relación con el área, la altitud y el tiempo de retención hidráulica. Acta Limnol. Bras. 13: 19-34.

Ramos, R . (2007). Modelación del Efecto del Viento sobre la Estructura Térmica del Embalse Porce II (Tesis). Facultad de Minas. Universidad Nacional de Colombia, Medellín.

Román, R . (2011). Caracterización espacio temporal de la estructura térmica de un embalse tropical poco profundo, abastecido parcialmente por bombeo. (Tesis de maestría). Facultad de Minas. Universidad Nacional de Colombia. Medellín.

Román, R ., Gómez, A. \& Toro, M. (2013). Efecto estacional de los afluentes en la estructura térmica de un pequeño embalse neotropical, La Fe-Colombia. Dyna. 177: 152-161.

Ríos, E.L. (2005). Caracterización ambiental de la ciénaga El Eneal, ubicada en la reserva San Guaré (municipio de San Onofre, Sucre) (Tesis de maestría). Facultad de Ingeniería. Universidad de Antioquia. Medellín.

Roldán, G . \& R uiz, E . (2001). Development of Limnology in Colombia. En R. G. Wetzel \& Gopal, B. (Eds.) Limnology in Developing Countries, 3. New Delhi: International Association for Limnology (SIL). p. 69-119.

Ruttner, F. (1953). Fundamentals of Limnology. $3^{\text {a }}$ Ed. Toronto: University of Toronto Press.

Soto, L .M . \& L ópez, C . (1996). Limnología física del embalse Socuy, Venezuela. Boletín del Centro de Investigaciones Biológicas. 30: 157-171.

Schiemer F. \& B oland, K.T. (Eds.) (1996). Perspectives in tropical limnology. Amsterdam: SPB Academic Publishing.

Straskaba, M., Tundisi, J.G. \& Duncan, A. (Eds.). (1993). Comparative reservoir limnology and water quality management. Dordrecht: Kluwer Academic Publishers.

Talling, J .F. (1963). Origins of stratification in an African Rift Lake. Limnol. Oceanogr. 8: 68-78.

Talling, J .F. (1965). Comparative problems of phytoplankton production and photosynthetic productivity in a tropical 
and a temperate lake. Mem. Ist. It. d’Idrobiol. (Suppl.). 18: 399-424.

Talling, J .F. \& Lemoalle, J. (1998). Ecological dynamics of tropical inland waters. Cambridge: University Press.

Tavera, R. \& Martínez-Almeida, V. (2005). Atelomixis as a possible driving force in the phytoplankton composition of Zirahuén, a warm-monomictic tropical lake. Hydrobiologia. 533: $199-208$

Thornton, K.W. (1990). Sedimentary processes. En K. W. Thornton, Kimmel, L.B. \& Payne, F.E. (Eds.). Reservoir limnology: Ecological perspectives. New York: Wiley. p. 43-69.

Tundisi, J .G ., M atsumura-Tundisi, T., Pontes, M .C ., Gentil, J .C . (1981). Limnological studies at quaternary lakes in eastern Brazil. I. Primary production of phytoplankton and ecological factors at lake Dom Helvécio. Rev. Bras. Bot. 4: 5-14.

Tundisi, J .G . \& M atsumura-Tundisi, T. (1984). Comparative limnological studies at three lakes in tropical Brazil. Verh. Internat. Verein. Limnol. 22: 1310-1314.

Tundisi, J .G . \& M atsumura-Tundisi, T. (2008). Limnología. São Paulo: Oficina de Textos.
Umaña, G., Haberyan, K.A., Shorn, S.P. (1999). Limnology in Costa Rica. En: Wetzel, R.G. \& Gopal, B. (Eds.). Limnology in Developing Countries, 2. International Association for Limnology (SIL), New Delhi. p. 33-62.

Urrego A . \& R amírez, J J (2000). Cambios diurnos de variables físicas y químicas en la zona de ritral del río Medellín, Colombia. Caldasia. 22: 127-141.

Vergara, N. (2005). Dinámica mensual de la capa superficial mezclada y la estabilidad térmica en la presa de un embalse tropical: Represa La Fe, El Retiro, Colombia (Tesis). Facultad de Ingenierías. Universidad de Antioquia. Medellín.

Villamizar, D. (1984). Algunas consideraciones sobre los riesgos de proliferación de plantas acuáticas en embalses. Revista Ainsa. 2: 23-29.

Villegas, B. (2004). Utilización de un Modelo Numérico para el Estudio de la Estructura Térmica del Embalse Porce II (Trabajo de grado). Facultad de Minas, Universidad Nacional de Colombia. Medellín. 